\title{
PREVENTION OF PREECLAMPSIA IN WOMEN WITH MULTIPLE PREGNANCY AFTER ASSISTED REPRODUCTION
}

DOl: $10.36740 /$ WLek202003116

\author{
Tamara G. Romanenko, Olha M. Sulimenko \\ DEPARTMENT OF OBSTETRICS AND GYNECOLOGY №1, SHUPYK NATIONAL MEDICAL ACADEMY OF POSTGRADUATE EDUCATION OF MINISTRY OF \\ HEALTH OF UKRAINE, KYIV, UKRAINE
}

\begin{abstract}
The aim: To reduce the frequency and severity of preeclampsia, to improve obstetrical and perinatal outcomes in women with multiple pregnancy after assisted reproduction by the development and implementation of the preventive algorithm with biochemical markers of endothelial dysfunction prospective analysis.

Materials and methods: Clinical and laboratory prospective analysis of 54 cases of twins in women, treated from infertility with assisted reproductive technologies (ART), using the method of intracytoplasmic sperm injection (ICSI) and frozen embryos transfer, have been made. It was proven, that women with multiple pregnancy are always in a high risk group of placental dysfunction (PD) and preeclampsia (PE). Depending on the treatment algorithm and preventive measures, 2 groups of patients were formed. Group I included 29 pregnant women with twins, managed in accordance with developed recommendations. We didn't find evidence-based European guidelines, that would recommend routine prescription of progesterone to improve chorion invasion and further placentation in such group of patients, but in order to prevent endothelial dysfunction and to decrease the incidence and severity of preeclampsia, placental abnormalities and intrauterine growth restriction (IUGR), we proposed the following algorithm: - micronized progesterone $200 \mathrm{mg}$ vaginally (PV), as soon as pregnancy was diagnosed by positive h(G-test, till 16 weeks of pregnancy, angioprotector diosmin $600 \mathrm{mg}$ once daily orally (PO), 2 courses: from 8 till 12 and from 16 till 20 weeks of gestation, antiaggregant - acetylsalicylic acid $150 \mathrm{mg}$ from 12 till 36 weeks of gestation. Group II included 25 pregnant women with twins after the same ART procedures, who have not received above mentioned treatment. Plasma concentrations of PIGF, sFIt- 1 and the ratio of sFIt-1/PIGF in the second trimester were investigated in both groups of women in order to assess the effectiveness of proposed preventive measures.

Results: Usage of preventive algorithm has shown the reduction of PE incidences in 26\%, PD in 28.1\%, IUGR in 35\%, prematurity by $23 \%$ and fetal distress in $18 \%$, that led to improvement of obstetrical and perinatal outcomes in I group of women with multiple pregnancies after ART-treated infertility, compared with group II $(p<0.05)$. The evaluation of PIGF, sFlt-1 plasma concentrations and the ratio of sFlt-1/PIGF in the second trimester of pregnancy reflected the effectiveness of our method in women with twins after ART. The level of PIGF in the study group was higher (186.5 \pm 12 vs $154.2 \pm 10.7 ; p<0.05)$, and the level of sFlt- 1 was lower (1523.1 \pm 40.3 vs $1835.3 \pm 33.6 ; p<0.05)$. Results of sFlt-1/PIGF ratio analysis in the I group also showed effectiveness of the method proposed ( $20.3 \pm 3.1$ vs $28.1 \pm 2.2 ; \mathrm{p}<0.05$ ).

Conclusions: The observed results suggest, that pregnant women with twins after ART-treated infertility are in a high-risk group of PE, PD and IUGR of one or both fetuses. Implementation of the proposed preventive algorithm allows to reduce the incidence of $\mathrm{PE}$, obstetrical and perinatal complications in this group of patients, and can be widely used in clinical practice. Evaluation and prospective assessment of biochemical markers, such as PIGF, sFlt-1 and sFlt-1/PIGF ratio, in the second trimester of pregnancy in the target groups may likely predict the development of PE and its severity.
\end{abstract}

KEY WORDS: preeclampsia, placental disfunction, multiple pregnancy, twins, acetylsalicylic acid, prevention, diosmin, placental growth factor (PIGF), soluble form of vascular endothelial growth factor (sFlt-1), PIGF / sFlt-1 ratio, assisted reproduction, endothelial dysfunction

Wiad Lek. 2020;73(3):494-497

\section{INTRODUCTION}

Annually, about 76,000 women and 500,000 children die due to preeclampsia (PE). Among women with complicated pregnancy (preeclampsia, gestational diabetes, gestational hypertension, intrauterine growth restriction (IUGR), placental detachment, etc.) there is 3.7-fold increase of hypertension, 2.2-fold increase of ischemic heart diseases, 2.7 -fold increase of cardio-vascular diseases in later life $[1,2]$.

According to ACOG, multiple pregnancy adue to assisted reproductive technologies is associated with an increased risk of preeclampsia (OR 2.7). A study of Opdahl S. et al [3] in the 2015 not only confirmed the high incidence of gestational hypertension, but also found that the incidence is higher in case after ART. Hypertensive disorders after
ART were observed in $5.9 \%$ of singletone pregnancies and in $12.6 \%$ of multiple pregnancies. In all cases, regardless of ART technology in singletone pregnancies the risk was the same, but it was the highest when using frozen embryos (risk 7.0\%, risk difference $1.8 \%$, CI 1.2-2.8). In twin pregnancies, the risk was also the highest when using frozen embryos (risk 19.6\%, risk difference $5.1 \%$, CI 3.7-7.1). In 2017 Storgaard M. et al [4] found that during pregnancy using the donor egg comparison with conventional pregnancies by ART, the risk of preeclampsia was the highest, with odds correction coefficient $\mathrm{OR}$, and was 2.11 (CI 1,42-3,15) in single pregnancies and OR 3,31 (CI $1,61-6,80)$ with twins.

Thus, according to statistics, the incidence of severe preeclampsia in multiple pregnancies is 3-4 times higher 
than in singletone pregnancies [5].

Numerous recent works are dedicated to search preeclampsia predictive markers. It should be noted at the same time that an activive research is made in order to find a universal test for determining the risk of complications during pregnancy, with the emphasis on molecular genetic markers. It has been repeatedly proven that angiogenesis is a key process in the formation of the placental vascular system. Angiogenic factors and their receptors are important regulators of placental vascular system $[6,7]$.

The complete formation of uterine arteries is regulated by proangiogenic (promoting the growth of the endothelium) and anti-angiogenic biological substances. The placental growth factor PlGF (placental growth factor) is mainly produced by trophoblast. During the pregnancy trophoblast and VEGF (vascular endothelial growth factor) belong to the first group. Their antagonist is soluble tyrosine kinase sFlt-1 (soluble fmslike tyrosinekinase). During the physiological pregnancy, there is a normal balance between these indicators - a certain amount of PlGF is synthesized, which binds to Flt-1 receptors located on the surface of the endothelial cells and promotes vasodilation of the uterine arteries. Excess of PlGF «utilized» by action of sFlt1. However, the process may be altered, and for some reason (not determined completely) promoting increased synthesis of sFlt-1 at normal amount of synthesized PIGF. This imbalance leads to increased binding of the placental growth factor by tyrosine kinase. Due to the lack of free PlGF, Flt-1 receptors do not receive the required amount of proangiogenic factor that causes vasoconstriction, the uterine arteries remain constricted, and blood flow is accelerated. This situation triggers a cascade of further pathophysiological changes that ultimately leads to serious health disorders in a pregnant woman organism and problems with fetal growth $[8,9,10]$.

According to published reports, antiangiogenic factors predominate in pregnant with twins. Interesting that the same group of pregnant women, but after ART presented with even more pronounced anti-angiogenesis $[6,11,12]$. The concentration of circulating biomarkers of sFlt- 1 and PIGF changes in women with PE even before its development and correlates with severity and early onset. In cases where PE subsequently developed, low levels of PIGF in the I trimester was observed and the level of sFlt- 1 can start to increse 5 weeks before its onset. Thus, in the II trimester, the ratio of sFlt- 1 and PIGF may serve as an informative marker of PE. Analysis of data from a multicenter prospective cohort study of pregnancies $(\mathrm{n}=$ 772) showed that in women with sigletone pregnancies the concentration of circulating sFltl and PIGF is lower than in multiple pregnancy [13].

The level of sFlt- 1 in case of physiological pregnancy begins to increase 5 weeks before the possible occurrence of the first clinical manifestations of PE, and further increases. There is a direct correlation between the level of sFlt-1, severity PE and the development of hypertension and proteinuria [9]. A decrease in PIGF level or increase of sFLT-1 / PIGF ratio during pregnancy is considered as a prognostic indicator of PE development $[9,14]$. In early and late forms of PE, the level of sFlt-1 in the mother's blood changes. Several studies have shown a 43 -fold increase of sFlt- 1 concentrations in case of early forms of $\mathrm{PE}$ and a 3-fold increase in case of late as compared to the concentration of sFlt- 1 on the background of physiological pregnancy. It is believed that the increase of sFlt- 1 level in the II trimester reliably corresponds to development of PE and is the most accurate marker of the development of its early form [15].

S. Verlohren et al. confirmed the high diagnostic value of Flt-1 / PIGF ratio in the period from 20 to 34 weeksof pregnancy as a marker of $\mathrm{PE}$ with a sensitivity of $95 \%$ and specificity of $94 \%$ [16].

All of the above is a clear justification for the relevance of the chosen research direction.

\section{THE AIM}

To reduce the frequency and severity of preeclampsia, to improve perinatal and obstetrical delivery outcomes in pregnant women with multiple pregnancy after in vitro fertilization due to improvement and implementation of the preventive measure algorithm and studying biochemical markers of endothelial dysfunction.

\section{MATERIALS AND METHODS}

According to this goal were conducted a prospective analysis of 54 twin pregnancies for the period from 2017 to 2019. Those pregnancies were achieved through assisted reproductive technology (ART), using the method of intracytoplasmic sperm injection (ICSI) and frozen embryos transfer. The usage of frozen embryos always leads to an increased risk of placental dysfunction (PD) and preeclampsia. Depending on the algorithm of treatment and preventive measures of complications, 2 groups were formed. Group I included 29 women pregnant with twins. They were offered the following algorithm: - micronized progesterone $200 \mathrm{mg}$ PV from the first day of pregnancy to 16 weeks of pregnancy; - angioprotector diosmin $600 \mathrm{mg}$ once a day, per os, 2 courses: 8 to 12 and 16 to 20 weeks of gestation; antiaggregant - acetylsalicylic acid $150 \mathrm{mg}$ from 12 to 36 weeks of pregnancy. Group II included 25 pregnant women with twins who didn't get the listed above treatment.

We propose the following algorithm of treatment and prophylactic to reduce the incidence of preeclampsia, obstetric and perinatal complications in pregnant women with multiple pregnancy after ART:

- hormone therapy: micronized progesterone $200 \mathrm{mg}$ per vaginum from the first day of pregnancy to 16 weeks;

- angioprotective therapy: diosmin $600 \mathrm{mg}$ per os 2 courses 8 to 12 weeks, 16 to 20 weeks;

- antiagregant therapy: acetylsalicylic acid $150 \mathrm{mg}$ for 12 to 36 weeks.

PIGF, sFlt-1, and sFlt-1 / PIGF ratios were studied in both trimesters in both groups. The effectiveness of the proposed treatment-and-prophylaxis algorithm was evaluated by perinatal and obstetric outcomes, plasma PIGF, sFlt-1, and sFlt-1 / PIGF ratios in the second trimester. Microsoft Excel XP and Statistica 6.0 Windows, methods of descriptive 
Table I. Peculiarities of pregnancy

\begin{tabular}{ccc}
\hline Complications & I group (n=29) & II group (n=22) \\
\hline Preeclampsia: & $10,4 \%(3)^{*}$ & $36,4 \%(8)$ \\
moderate & $6,9 \%(2)$ & $18,2 \%(4)$ \\
severe & $3,5 \%(1)$ & $18,2 \%(4)$ \\
\hline Placental dysfunction: & $17,3 \%(5)^{*}$ & $45,5 \%(10)$ \\
\hline IUGR (degree): & $10,4 \%(3)$ & $45,5 \%(10)$ \\
II & $6,9 \%(2)$ & $13,6 \%(3)$ \\
III & $3,5 \%(1)$ & $22,8 \%(5)$ \\
\hline Fetal distress & - & $9,1 \%(2)$ \\
\hline Preterm labor & $13,8 \%(4)$ & $31,8 \%(7)$ \\
\hline Before 34 weeks of gestation & $17,3 \%(5)$ & $36,4 \%(8)$ \\
After 34 weeks of gestation & $6,9 \%(2)$ & $22,8 \%(5)$ \\
\hline PANSP & $10,4 \%(3)$ & $13,6 \%(3)$ \\
\hline
\end{tabular}

Note: ${ }^{*} p<0.05$ compared to group II indicators

Table II. Features of newborns condition

\begin{tabular}{ccc}
\hline Complications & I group (n=58) & II group(n=44) \\
\hline Hypoxic-ischemic CNS injury & $6,9 \%(4) *$ & $15,9 \%(7)$ \\
\hline Disorders of adaptation & $17,2 \%(10) *$ & $38,6 \%(17)$ \\
\hline Transfer of neonates to 2ndstage of treatment & $6,9 \%(4) *$ & $25 \%(11)$ \\
\hline
\end{tabular}

Note: ${ }^{*} p<0.05$ compared to group II indicators

Table III. Biochemical markers of preeclampsia in the second trimester

\begin{tabular}{ccc}
\hline Value & I group $(\mathbf{n}=\mathbf{2 9})$ & II group (n=22) \\
\hline PIGF & $186,5 \pm 12,6$ & $154,2 \pm 10,7$ \\
\hline sFlt-1 & $1523,1 \pm 40,3$ & $1835,3 \pm 33,6$ \\
\hline sFlt-1/PIGF & $20,3 \pm 3,1^{*}$ & $28,1 \pm 2,2$ \\
\hline
\end{tabular}

Note: ${ }^{*}<<0,05$ compared to Group II values

statistics and correlation analysis were used for statistical processing.

\section{RESULTS}

Analysis of the clinical course and termination of pregnancy showed significantly better results in group I ( $p<0.05$ compared to group II indicators) (Table I).

The incidence of preeclampsia in group I was only in $10.4 \%$ cases (3) versus in group II $36.4 \%$ cases (8) (group I had 1 case $(3.5 \%)$ of severe preeclampsia and $2(6.9 \%)$ cases of moderate preeclampsia; in group II moderate and severe preeclampsia were observed in $18.2 \%$ (4) and $18.2 \%$ cases (4) respectively). Placental dysfunction was diagnosed in $17.3 \%$ (5) of group I cases and in $45.5 \%$ (10) of group II. Intrauterine growth restriction was less common in Group I - $10.4 \%$ (3) (I degree 6.9\% (2), II degree in 3.5\% (1), no cases of III degree in comparison with $45.5 \%$ cases (10) in Group II (I degree 13.6\% (3), II degree 22.8\% (5), III degree $9.1 \%$ (2). Fetal distress was diagnosed in 4 pregnant women $(13.8 \%)$ of group I, and in 7 women of group II (31.8\%). We recorded $5(17,3 \%)$ preterm labor (before 34 weeks of gestation - 2(6,9\%); after 34 weeks of gestation
- $3(10,4 \%)$ in the group where the proposed prevention regimen was applied. In the group where the conventional regimen was applied there were 8 cases $(36.4 \%)$ of preterm labor(before 34 weeks of gestation - $5(22.8 \%)$; and 3 cases (13.6\%) after 34 weeks of gestation. Placental abruption was diagnosed in $9.1 \%$ (2) among group II, and in group I no such cases were recorded. The peculiarityof newborns' status are shown in table II.

Hypoxic-ischemic injuries occurred in 4 (6.9\%) children of group I and in 7 (15.9\%) children of group II. Disorders of adaptation were observed significantly less in the group using the proposed scheme prevention - $10(17.2 \%)$ cases, compared to group II - 17 (38.6\%) cases. Transfer to the second stage of treatment required $4(6.9 \%)$ neonates in group I and $11(25 \%)$ neonates in group II.

\section{DISCUSSION}

An investigation of plasma concentration of PlGF, sFlt-1, and the sFlt-1 / PlGF ratio in the second trimester of pregnancy confirmed the effectiveness of treatment and preventive measures (Table III), namely: the level of PlGF in the study group was higher $(186.5 \pm 12.6$ opposite to $154.2 \pm 10.7$, 
$\mathrm{p}<0,05)$ and the level of sFlt- 1 lower $(1523.1 \pm 40.3 \mathrm{vs.} 1835.3 \pm$ 33.6,p $<0,05)$.The results of the sFlt- $1 /$ PlGF ratio in the study group also indicated a better effect of the proposed prophylaxis method $(20.3 \pm 3.1$ vs. $28.1 \pm 2.2, \mathrm{p}<0,05)$.

Usage of our advanced preventive measures algorithm can reduce the incidence of preeclampsia by $26 \%$, placental dysfunction by $28.1 \%$, preterm labors by $23 \%$, fetal distress by $18 \%$, fetal growth retardation by $35 \%$, which together improves obstetric and perinatal outcomesin case of multiple pregnancy after ART.

\section{CONCLUSIONS}

Thus, the results of the study indicate that pregnant women with twins achieved through ART are at high risk of preeclampsia, placental dysfunction, and fetal growth retardation. Modern scientific methods of prevention of obstetric and perinatal complications are needed in order to solve this problem. The proposed algorithm of modern diagnostic and preventive measures allowes to significantly reduce the incidence of preeclampsia, obstetrical and perinatal complications in this group of pregnant women and can be used in practical public health care. Determination of the biochemical markers, such as PlGF, sFlt-1 and sFlt-1 / PIGF ratio in the second trimester may be likely to predict the development of preeclampsia and its severity.

\section{REFERENCES}

1. WHO recommendations for Prevention and treatment of pre-eclampsia and eclampsia, 2011.

2. National Institute for Health and Care Excellence (2019) Hypertension in pregnancy: diagnosis and management. NICE guidelines [NG133], June 25.

3. OpdahlS, Henningsen A, Tiitinen A et al. Risk of hypertensive disorders in pregnancies following assisted reproductive technology: a cohort study from the CoNARTaS group. Human Reproduction, 2015;7:1724-1731.

4. Storgaard, M., Loft, A., Bergh, C. et al. Obstetric and neonatal complications in pregnancies conceived after 0ocyte donation - a systematic review and meta-analysis. BJOG. 2017;124:561-572.

5. S.V. Barinov, E.V. Rogova, T.V. Kadtsyina. Prognozirovanie riska tyazheloy preeklampsii pri mnogoplodnoy beremennosti i puti ee profilaktiki. Vestnik RUDN, seriya Meditsina, 2015;1.

6. Lee M.S. Angiogenic markers in pregnancies conceived through in vitro fertilization. Am J Obstet. Gynecol. 2015;213,212:1-8

7. Anand S, Bench Alvarez T, Johnson W et al. Serum biomarkers predictive of preeclampsia. Biomark Med. 2015;9(6):563-575. D0I: 10.2217/ bmm.15.21.

8. Lecarpentier É. et al Placental growth factor (PIGF) and sFlt-1 during pregnancy: physiology, assay and interest in preeclampsia.. AnnBiolClin (Paris). 2016;Jun 1;74(3);259-267.

9. Stepan H., Schaarschmidt W., Jank A. et al. Use of angiogenic factors (sFlt-1/PIGF ratio) to confirm the diagnosis of preeclampsia in clinical routine: first experience. Z Geburtshilfe Neonatol. 2010;214(6):234238. DOI: $10.1055 / \mathrm{s}-0030-1262827$.
10. Stepan H. et al. Implementation of the sFlt-1/PLGD ratio for prediction and diagnosis of preeclampsia in in singleton pregnancy: implications for clinical practice. Ultrasound Obstet. Gynecol. 2015. Mar; 45(3):241-6.

11. Tsiakkas A. et al. Serum soluble fms-like tyrosine kinase- 1 in the three trimesters of pregnancy: effects of maternal characteristics and medical history. UK, Ultrasound Obstet. Gynecol.2015 May 45(5).

12. Sánchez 0 . et al. First trimester serum angiogenic/antiangiogenic status in twin pregnancies: relationship with assisted reproduction technology. Spain, Hum. Reprod. 2012:27(2).

13. Boucoiran L., Thissier-Levy S., Wu Y.r MD et al. Risks for Preeclampsia and Small for Gestational Age: Predictive Values of Placental Growth Factor, Soluble fms-like Tyrosine Kinase-1, and Inhibin A in Singleton and Multiple-Gestation Pregnancies. Am. J. Perinatal. 2013;30:607-612.

14. Kenny L.C., BlackM.A., Poston L. et al. Early pregnancy prediction of preeclampsia in nulliparous women, combining clinical risk and biomarkers: The Screening for Pregnancy Endpoints (SCOPE) international cohort study. Hypertension. 2014;64(3):644-652.

15. Cox A.G., Marshall S.A., Palmer K.R., Wallace E.M. Current and emerging pharmacotherapy for emergency management of preeclampsia. Expert Opinion on Pharmacotherapy. 2019;20(6):701-712.

16. Verlohren S., Herraiz I., Lapaire 0 . New gestational phase-specific cutoff values for the use of the soluble fms-like tyrosine kinase-1/placental growth factor ratio as a diagnostic test for preeclampsia. Hypertension. 2014;63(2):346-352.

\section{ORCID and contributionship:}

Tamara G. Romanenko - 0000-0003-0157-6223 A,B,C,D

Olha M. Sulimenko - 0000-0003-1948-8387 A,B,C,D,F

\section{Conflict of interest:}

The Authors declare no conflict of interest.

\section{CORRESPONDING AUTHOR Olha M. Sulimenko}

Department of Obstetrics and Gynecology №1, Shupyk National Medical Academy of Postgraduate Education of Ministry of Health of Ukraine 9 Dorogozhytska street, Kyiv, Ukraine, 04112

tel: +380509785166

e-mail: sulimenko.olga5@gmail.com

Received: 17.01.2020

Accepted: 05.03.2020

A - Work concept and design, B - Data collection and analysis, C - Responsibility for statistical analysis, D-Writing the article, $\mathbf{E}$-Critical review, $\mathbf{F}$ - Final approval of the article 